\title{
Observing prioritization effects on cognition and gait: the effect of increased cognitive load on cognitively healthy older adults' dual-task performance
}

Article

Accepted Version

Creative Commons: Attribution-Noncommercial-No Derivative Works 4.0

MacLean, L. M., Brown, L. J.E., Khadra, H. and Astell, A. J. (2017) Observing prioritization effects on cognition and gait: the effect of increased cognitive load on cognitively healthy older adults' dual-task performance. Gait and Posture, 53. pp. 139-144. ISSN 0966-6362 doi:

https://doi.org/10.1016/j.gaitpost.2017.01.018 Available at https://centaur.reading.ac.uk/69275/

It is advisable to refer to the publisher's version if you intend to cite from the work. See Guidance on citing.

To link to this article DOI: http://dx.doi.org/10.1016/j.gaitpost.2017.01.018

Publisher: Elsevier Ltd

All outputs in CentAUR are protected by Intellectual Property Rights law, including copyright law. Copyright and IPR is retained by the creators or other copyright holders. Terms and conditions for use of this material are defined in the End User Agreement. 


\section{www.reading.ac.uk/centaur}

\section{CentAUR}

Central Archive at the University of Reading

Reading's research outputs online 


\section{Abstract}

Previous studies exploring the effects of attention-prioritization on cognitively healthy older adults' gait and cognitive dual task (DT) performance have shown DT cost in gait outcomes but inconsistent effects on cognitive performance, which may reflect task difficulty (the cognitive load). This study aimed to identify whether changing the cognitive load during a walking and counting DT improved the challenge/sensitivity of the cognitive task to observe prioritization effects on concurrent gait and cognitive performance outcomes. Seventy-two cognitively healthy older adults (Mean $=73$ years) walked $15 \mathrm{~m}$, counted backwards in $3 \mathrm{~s}$ and 7s as single tasks (ST), and concurrently walked and counted backwards as DTs. Attention-prioritization was examined in Prioritizing Walking (PW) and Prioritizing Counting (PC) DT conditions. Dual-task performance costs (DTC) were calculated for number of correct cognitive responses (CCR) in the counting tasks, and step-time variability and velocity in the gait task. All DT conditions showed a benefit (DTB) for cognitive outcomes with trade-off cost to gait. In the Serial 3s task, the cognitive DTBs increased in PC over the PW condition $(p<.05)$, with a greater cost to walking velocity $(p<.05)$. DT effects were more pronounced in the Serial 7s with a lower cognitive DTB when PC than when PW, $(p<.05)$ with no trade-off increase in cost to gait outcomes $(p<.05)$. The findings suggest that increased cognitive load during a gait and cognitive DT produces more pronounced gait measures of attention-prioritization in cognitively healthy older adults. A cognitive load effect was also observed in the cognitive outcomes, with unexpected results. 
Introduction

Despite appearing automatic, older adults find walking requires some degree of attention and the addition of a concurrent cognitive task increases demands on their attentional resources [1-2]. This can compromise gait in the form of slower walking speed and/or greater variability in step-time or step-length between steps. The additional demand on attention is important, as the ability to carry out concurrent activities is essential to independent-living [3], and failing to prioritize the right task at the right moment presents risks, such as falls [4], in older adults' daily life[5]. Measures of concurrent activity are also predictive of future cognitive decline [6]. Therefore, sensitive measurement of the ability to allocate attention could be a useful tool for assessing older adults' current cognitive function and risk of future difficulties.

One method for investigating allocation of attention during concurrent activity uses the dual task (DT) paradigm [7], which compares performance on two single tasks carried out separately with performance when carrying out the two tasks concurrently. The DT impact is calculated as either a dual-task cost (DTC) or dual-task benefit (DTB) for one or both concurrent tasks, where DTC indicates by how much each task performance declines in the DT condition or improves (DTB).

In DT the type of cognitive task has been shown to have different effects; step-time walking variability appears to be affected more by an arithmetic task (e.g. counting backwards, subtracting 7s) than a verbal fluency task [8]. By increasing the demand for mental effort required in each task (e.g. subtracting $7 \mathrm{~s}$ requires more attentional resources than subtracting 3s), the difficulty of these arithmetic tasks can be varied in order to create distinct levels of cognitive difficulty [9]. 
When faced with a demanding DT situation such as concurrent walking and counting, it is thought healthy older adults prioritize gait over cognition (the so-called 'posture first strategy') [10]. This involves flexible attention-allocation between the two tasks in order to ensure gait stability, which often results in more cognitive errors. It has been proposed that explicitly instructing participants to prioritize one task over the other e.g. prioritise gait over cognition, during the DT, permits observation of which activity older adults actually allocate their attention to [11]. Measuring the effects of prioritization instructions on both gait and cognitive outcomes extends DTC and DTB findings to examine changes in the pattern of attention-allocation.

Prioritization instructions have consistently shown effects on gait outcomes. For example, when instructed to prioritize walking, velocity increases (i.e. faster walking) compared to when the cognitive task is prioritized $[5,11-13]$. Similarly, gait variability is more stable under specific instructions to prioritize gait [13]. In contrast, prioritization of cognition has been less consistent. For example, healthy older adults instructed to prioritize reciting words starting with a given letter produced more verbal responses but less stable gait [11]. However, when asked to prioritize reciting alternate letters of the alphabet, Verghese and colleagues reported no significant changes in the cognitive task, although walking speed did become slower [12]. Furthermore, Siu and colleagues reported that neither cognitive responses nor gait benefitted from participants' prioritization of a cognitive task [4] The discrepancies may be due to differences in the difficulty of the cognitive tasks used, that is the 'cognitive load' of each task may vary. This variability may indicate that to observe effects on gait and cognition, the cognitive task must be of optimal difficulty. To our knowledge, no studies have investigated concurrent gait and cognitive activity by varying the cognitive loads (for task difficulty) and changing prioritization instructions to observe 
effects on both gait and cognitive outcomes. To test the hypothesis that changing the cognitive load increases sensitivity of prioritization, we measured the effects of prioritization under different levels of cognitive load. We predicted that the DT gait costs would be greater when cognitively healthy older adults were instructed to prioritize counting rather than when instructed to prioritize walking, i.e. reduced walking speed and increased step-time variability. We also predicted that the magnitude of DTCs for gait would increase with task difficulty, that is greater DTCs would be observed for gait outcomes in the more difficult cognitive task. In addition, if the ability to observe DT effects on the pattern of cognition and gait outcomes, under explicit prioritization instructions, does depend on the level of cognitive load applied, then we also predicted that prioritization effects on cognitive responses would also be greater under conditions of high cognitive load (the Serial 7s task) than low cognitive load (Serial 3s). That is, there would be greater DTCs for cognitive responses in the Serial 7s than in the Serial 3s task.

Methods

Design

A repeated-measures design with independent variables of cognitive load and explicit prioritization instruction was used. The two levels of cognitive load were: counting backwards in 3s (low cognitive load: hereafter Serial 3s); and counting backwards in 7s (high cognitive load: Serial 7s). The different prioritization instructions were: prioritizing walking (PW); and prioritizing counting (PC). The dependent variables were: Dual-Task Cost (DTC) for cognitive performance measured as number of correct cognitive responses (CCR) per second, and gait measured by both step-time variability and velocity. Step-time variability 
was expressed as coefficient of variation (CV; standard deviation divided by mean) whilst velocity was calculated as time taken (in seconds) to walk $15 \mathrm{~m}$.

\section{Participants}

A G-power analysis with a small effect size, was carried out, and the highest number of participants (65) at a power of 0.9 was selected. To address attrition 72 were recruited. Forty-three women and 29 men, with a mean age of 73 (5.7 SD) years, were recruited from the local community in and around St Andrews, Scotland. Inclusion criteria were: age 65 years or over; living independently; able to walk unassisted; English as the first language. Exclusion criteria were: Mini-Mental State Examination (MMSE) [14] score of 27 or above (adjusted in one case for age and education [22]); or self-reported presence of a chronic physical or mental illness that might adversely affect gait or cognitive function.

\section{Equipment}

The 'Bigfoot' footswitch system was developed to measure walking velocity and step-time variability by capturing the time between the on/off contacts on the footswitches attached to each of the participant's heels [15]. Bigfoot records successive heel-strikes, analyzes the time it takes participants to produce each step and calculates the step-time variability (CV) and velocity for each walking condition. Bigfoot was validated manually against 'off -the shelf', state of the art video recording devices of gait measurement [15]. 


\section{Materials}

Self-report measures were used to collect demographic and background characteristics: age, total years in education, weekly number of IADLs, physical exercise, cognitive activity, alcohol consumption (units), tobacco use history (current, past, never), visual impairment, hearing impairment, existing chronic medical conditions, number of regular medications, number of falls and hospitalisations in the previous year, satisfaction with current health status. Body Mass Index (BMI) was also calculated. Standardised measures included: The Beck Depression Inventory-II (BDI)[16]; a self-report screen for depression; National Adult Reading Test [17] to provide estimated premorbid IQ; general cognition MMSE [14], memory [digit span; 18-19], processing speed [SDMT; 20] and Executive Functions (Trail Making A\&B; [21];Table 1).

Table 1 here

\section{Procedure}

After completing the standardized measures the participants completed seven conditions: one single walking trial, two single counting trials, and four DT prioritization trials. In the single walking condition, participants walked along a 15-metre course (with 1 meter added to the start and end for acceleration and deceleration) at their own pace. In the single counting conditions, participants were seated and recorded counting backwards, subtracting either 3s (Serial 3s) or 7s (Serial 7s) from a given number for one minute. The DT conditions were walking along the same $15 \mathrm{~m}$ walkway, and counting (Serial $3 \mathrm{~s}$ and $7 \mathrm{~s}$ ) 
prioritizing either walking or counting. At the start of each DT condition the participants were asked to walk and count backwards in either $3 \mathrm{~s}$ or $7 \mathrm{~s}$, and instructed to either concentrate more on walking than counting (PW) or more on counting than walking (PC). The ST conditions were counter-balanced and alternated and the DT conditions were randomized. The start-number for each ST and DT trial was a randomly chosen 3-digit number between 102 and 298. Each ST and DT counting task started with a different 3-digit number, with no two digits the same in any of the numbers. Each condition had the same start number for each participant. There were no specific instructions for mistakes occurring in the DT counting task or hesitations in the DT walking performance. All of the ST and DT conditions were video-recorded using a Sony Cybershot 7.2 camera, which also recorded participants' counting aloud.

\section{Scoring of Correct Cognitive Responses (CCR)}

The baseline CCR score in the ST condition was calculated by dividing the number of correct responses enumerated by the time taken (60 seconds) to produce a response rate per second. This number was then multiplied by the ratio of correct responses to total responses, to take error into account, with higher CCR scores indicating better cognitive performance. If a participant made a mistake in their subtraction, that response was scored as an error and the next response they gave was scored either correct or wrong in relation to that 'error'. Thus errors were only penalized once.

The CCRs scores in the four DT prioritizing conditions (3PW, 7PW, 3PC and 7PC) were first calculated as the number of correct responses given in the DT, divided by the time taken for 
each individual DT condition and this figure was then multiplied by the ratio of correct responses to total responses, to adjust for errors. The cost (DTC) or benefit (DTB) in each condition was calculated by subtracting the DT from the ST score.

\section{Statistical analyses}

Gait step-time variability data were not normally distributed, and so were transformed using a Log10 transformation equation, and then analyzed using parametric tests. The effects of load and prioritization on gait and cognitive outcomes for all four DT conditions were analysed with a series of $2 \times 2$ repeated measures ANOVAs. Where significant interactions were found, t- tests with Bonferroni corrections, were used to investigate differences in specific pairs of outcomes. Alpha levels of significance were set at 0.05 level and effect sizes were reported as $\eta^{2}$ or $r$. All data were analyzed using SSPS version 21.0 for Windows (2010).

Results

\section{Participants' characteristics}

One participant scored below MMSE 27, but was inside normal limits age and education adjustments. Three participants registered mild depression $(B D I>14)$ and two of these scored below average on the SDMT $(<37.4)$. Removing their data had no impact on the direction or significance of the results, therefore, the data for all 72 participants are reported. All other standardized cognitive test scores for the complete cohort were within 
the normal range and indicated that this was a group of cognitively healthy older adults (Table 1).

Table 2. here

The participants' self-reported health, mood and lifestyle characteristics are summarized in Table 2. The group mean BMI was overweight, and over a third of the cohort took more than 3 medications per week. However, the participants exercised regularly and $86 \%$ were satisfied with their current health status.

Table 3 here

Mean (M) and standard deviation (SD) for ST and DT, and DTCs and DTBs for the four DT conditions are shown in Table 3. Repeated measures ANOVA were performed on all mean outcome scores to assess the effect of changing cognitive load on the patterns of prioritization of attention under specific prioritizing instructions (Table 4.)

Table 4. here 
The mean DTC and DTB outcomes and patterns of trade-off between the concurrent walking and counting tasks under each DT prioritizing condition are shown in Figure 1. DT values for CCR and velocity have been reversed in this figure so that negative values always indicate DTCs, and positive values always indicate DTBs. Negative step-time variability scores indicate a DTC.

Fig. 1 here

\section{Gait Outcomes}

For step-time variability, there was a main effect of cognitive load $(p<.01)$, with higher DTC in the Serial 7s task (Table 4). There was also a main effect of prioritizing instructions, with the PC condition producing greater DTC in step-time variability $(p<.01)$. In addition, there was a significant load $\mathrm{x}$ prioritization interaction $(p<.01)$. T- tests for step-time variability in the Serial 3s task found no significant difference between the PW (Mean $=-.013$ ) and the PC (Mean $=-.017)$ conditions, but for the Serial 7s task there was a greater DTC in the PC condition (Mean $=-.049$ ), compared with the PW condition (Mean $=-.016 ; p<.001$ ). For velocity, there was also a main effect of cognitive load $(p<.001)$ with greater DTC in the Serial 7s task and a main effect of prioritization $(p<.01)$ but there was no significant load $\mathrm{x}$ prioritization interaction under the higher cognitive load (Table 4). 
All DT conditions showed a benefit for CCRs with a trade-off cost to gait (Table 4). For correct cognitive responses (CCRs) there was a main effect of load (higher vs. lower; $p<.05$ ). That is, whilst both cognitive loads produced dual-task benefits, these DTBs were significantly smaller in the Serial 7s (Mean $=-.021)$ task than in the Serial 3s (Mean $=-.131 ; p$ $<.05)$. No main effect of prioritization was found $(p>.05)$, but there was a significant load $\mathrm{x}$ prioritization interaction, which took different directions depending on the cognitive load ( $p$ $<.01)$. T- tests showed that there was a greater DTB in the Serial 3s PC condition ( $M=-.139)$ than in the Serial 3s PW condition (Mean $=-.085 ; p<.01$ ). However, in the Serial 7s PC condition, contrary to predictions, when cognition was prioritized (Mean $=-.021$ ), the DTB was non-significant (relative to the ST; $p>.05$ ) and smaller relative to Serial 7S PW (Mean = .085). However, the cognitive performance for Serial 7s actually improved (a bigger and statistically significant DTB was produced) when walking was prioritized (Mean $=-.085 ; p$ $<.001)$. This latter DT cognitive benefit, when walking was prioritized, was achieved at no relative cost to either gait variability or velocity giving rise to an 'optimal performance' (Figure 1.).

\section{Discussion}

This study investigated the prioritization effect on both gait and cognitive outcomes of changing the cognitive load during a walking and counting DT. The key findings were that increasing cognitive load adversely affected both gait outcomes (velocity and step-time variability). Prioritization effects were only seen on step-time variability under higher cognitive load, which might explain why a previous prioritization study with only one level of cognitive difficulty, found prioritization effects for walking velocity, but not step-time 
variability [12]. For walking velocity, as opposed to step-time gait variability, our results suggest that, under both low and high cognitive loads, prioritization effects on gait reflect specific prioritization instructions. That is, gait became slower when the cognitive load was increased and slower when attention was directed away from walking onto counting in Serial 7s, but the difficulty of the cognitive task did not affect walking velocity when attention-prioritization instructions were changed (the same pattern was observed for both Serial 3s and Serial 7s). However, patterns of attention-allocation in gait variability outcomes change, contrary to expectations, when the cognitive load increases.

In respect of cognition, there were DT benefits (rather than costs or no effect) for all four DT counting performances which suggests that there is not necessarily a cost to cognition when attentional demand is increased in concurrent activity. (One might expect that this would come with a trade-off cost of DTCs in both walking velocity and gait step-time variability.) (Fig. 1). DTBs for the cognitive task have previously been explained as possibly due to the synchronicity of concurrent tasks [23] or to healthy participants having sufficient postural reserve to allocate resources to the cognitive task when there is no significant risk of falling [11]. This finding of DTB is an encouraging sign that healthy older adults can allocate attention when they have to complete complex tasks.

A second unexpected finding was that, under the higher cognitive load (Serial 7s), the DTB in cognitive responses was smaller when counting was prioritized and greater when walking was prioritized. However, as expected under the lower cognitive load (Serial 3s), the DTB in cognitive responses was greater when counting was prioritized and smaller when walking was prioritized. Under the higher cognitive load, although there was a small DTB when instructed to prioritize counting, it was not statistically significant, unlike the other cognitive 
DTBs, and gait worsened (relative to the all other prioritizing conditions). This indicates that, instead of prioritizing counting as instructed, our participants apparently prioritized gait. Given that they could perform well in both walking and counting in the higher cognitive load when instructed to prioritize walking, they clearly had sufficient DT attentional resources to adhere to a specific instructional set, but they did not use these resources to allocate attention to the cognitive task when the cognitive load was increased. We suggest that these healthy older adults prioritized gait over the cognitive task (in the PC condition), to secure gait stability, which was under threat from a heavier demand on their attentional resources. This supports previous findings that healthy older adults will adapt their walking in response to high cognitive demands [24]. Our results also provide further evidence for the 'posture-first' strategy whereby, if the cognitive load during a DT presents a threat to gait security, 'postural control would be the first priority for attentional resources' [10] (p. 238).

\section{Limitations}

The participants were generally in good physical and cognitive health and of relatively high socio-economic status, which may reduce the generalizability of the results. The authors also acknowledge that there is some debate about the ideal walk length from which to obtain sufficient steps to calculate gait variability. Future studies might include extended walking tests.

\section{Conclusions}

Our findings offer an approach to observing older adults' attention-prioritization during a DT when cognitive load changes. This has potential for illuminating attention-allocation during 
other complex daily tasks that could be of interest to both researchers and clinicians. This methodology could also be useful for assessing potential future cognitive change. 3012 words (including 15 words of instructions to insert tables/figure)

\section{References}

[1] Woollacot M and Shumway-Cook A (2002) Attention and the control of posture and gait: a review of an emerging area of research. Gait and Posture, 16(1): 1-14

[2] Hausdorff JM (2005) Gait variability: methods, modeling and meaning. Journal of NeuroEngineering and Rehabilitation2(19) doi:10.1186/1743-0003-2-19

[3] Sanders A E, et al. (2008) "Egocentric and Exocentric Navigation Skills in Older Adults." The Journals of Gerontology Series A: Biological Sciences and Medical Sciences 63(12): 13561363

[4] Woollacott, M. and A. Shumway-Cook (2002). "Attention and the control of posture and gait: a review of an emerging area of research." Gait Posture 16: 1-14

[5] Siu K-C, Chou L-S, Mayr U, van Donkelaar PandWoollacott MH. (2008). Does Inability to Allocate Attention Contribute to Balance Constraints During Gait in Older Adults? Journal of Gerontology: MEDICAL SCIENCES, 63A(12), 1364-1369.

[6] Springer, S., et al. (2006). "Dual-tasking effects on gait variability: the role of aging, falls, and executive function." Mov Disord 21: 950 - 957.

[7] Della Sala S, Baddeley A, Papagno C, Spinnler H (1995) Dual-task paradigm: A means to examine the central executive. Annals New York Academy of Sciences 769: 161-172 
[8] Beauchet, O., Dubost, V., Aminian, K., Gonthier R., Kressig, R.W. (2005). "Dual-taskrelated gait changes in the elderly: does the type of cognitive task matter?" Journal of Motor Behavior 37(4): 259-264.

[9] Hausdorff J. M., Schweiger A., Herman T., Yogev-Seligmann G., \& Giladi N. (2008). Dualtask decrements in gait: contributing factors among healthy older adults. The Journals of Gerontology Series A: Biological Sciences and Medical Sciences, 63(12), 1335-1343.

[10] Shumway-Cook A, Woollacott, M, Kern, K. A, and Baldwin M (1997) The Effects of Two Types of Cognitive Tasks on Postural Stability in Older Adults With and Without a History of Falls. The Journals of Gerontology Series A: Biological Sciences and Medical Sciences, 52A(4) M232-M240 doi:10.1093/gerona/52A.4.M232

[11] Yogev-Seligmann G, Rotem-Galili Y, Dickstein R, Giladi N, Hausdorff J M (2012a). Effects of explicit prioritization on dual task walking in patients with Parkinson's disease. Gait \& Posture 35(4): 641-646

[12] Verghese J, Kuslansky G, Holtzer R, Katz M, Xue X, Buschke H, andPahor M (2007) Walking While Talking: Effect of Task Prioritization in the Elderly Archives of Physical Medicine and Rehabilitation 88(1) 50-53 doi: 10.1016/j.apmr.2006.10.007

[13] Yogev-Seligmann G, Rotem-Galili Y, Mirelman A, Dickstein R, Giladi N and Hausdorff J M (2010). How Does Explicit Prioritization Alter Walking During Dual-Task Performance? Effects of Age and Sex on Gait Speed and Variability. Physical Therapy 90(2):177-186. doi: $10.2522 / p t j .20090043$

[14] Folstein M, Folstein S, \& McHugh P (1975) "Mini-mental state". A practical method for grading the cognitive state of patients for the clinician. J Psychiatr Res 12: 189 198 
[15] Maclean L. M, Brown L J E, Astell A J (2014) The Effect of Rhythmic Musical Training on Healthy Older Adults' Gait and Cognitive Function. The Gerontologist doi:10.1093/geront/gnt/050

[16] Beck AT, Steer RA, Brown GK (1996) Manual for the Beck Depression Inventory-II. San Antonio, TX: Psychological Corporation

[17] Nelson H E (1982) National Adult Reading Test Windsor. Smith A (1982) Symbol Digits Modalities Test, Los Angeles Western Psychological Services

[18]. Thorndike R L, Hagen E P, Sattler J M (1987) The Stanford-Binet Intelligence Scale (4th ed.) Chicago, Riverside.

[19] Wechsler D (1987) Wechsler Memory Scale - Revised (WMS-R) San Antonio, TX: Psychological Corporation.

[20] Smith A (1982) Symbol Digits Modalities Test, Los Angeles Western Psychological Services.

[21] Reitan R M, Wolfson D (1993) The Halstead-Reitan Neuropsychological Test Battery: Theory and clinical interpretation ( 2nd ed.) Tucson AZ: Neuropsychology Press.

[22] Lezak MD, (1995) Neuropsychological Assessment (3rd ed) Oxford : Oxford University Press

[23] Beauchet, O., et al. (2010). "Decrease in gait variability while counting backward: a marker of "magnet effect"?" Journal of Neural Transmission 117(10): 1171-1176.

[24] Li K Z H, Abbud G A, Fraser S A and DeMont R G (2013).Successful adaptation of gait in healthy older adults during dual-task treadmill walking. Aging, Neuropsychology, and Cognition 19(1-2);150-167 
Figure legend

Fig.1 Dual Task Costs and Benefits for step-time variability (CV), Velocity and CCRs for Serial 3s and Serial 7s cognitive tasks under PW and PC prioritizing conditions.

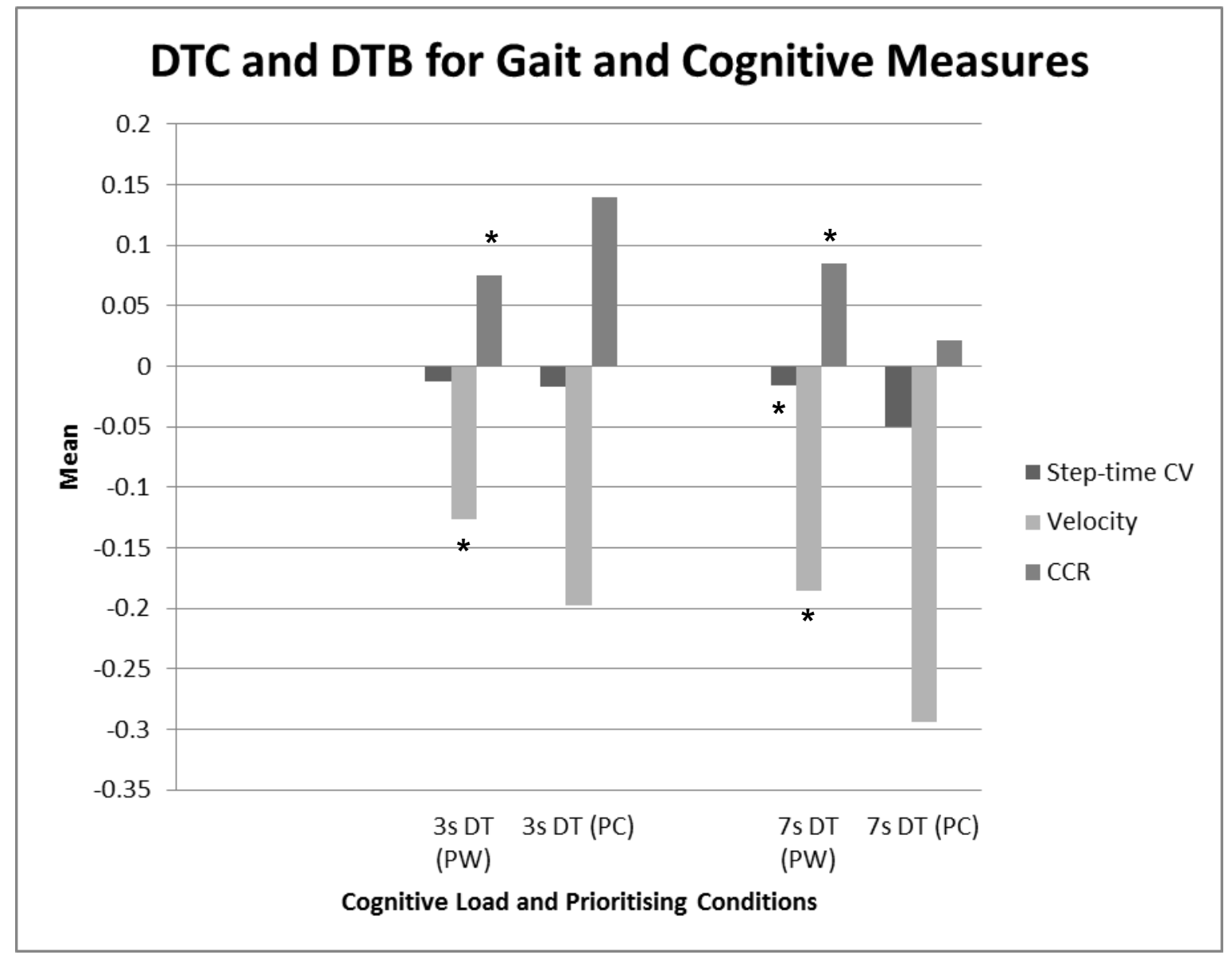

Note: Bars above 0 indicate a DTB and bars below 0 indicate a DTC. Values have been reversed for Velocity and CCRs for ease of reporting

*significant difference in DTC or DTB between PW and PC conditions, CV $(p<.001)$, Velocity $(p<.001)$ and CCRs $(p<.01)$ 
Table 1. Standardized neuropsychological test battery

\begin{tabular}{|c|c|c|c|c|c|c|}
\hline Test & Cognitive function & Scoring & Mean & SD & Range & Comments \\
\hline Mini Mental-State & General cognitive & 30-point scale & 28 & \pm 1.33 & $24-30$ & Cut-off 27 or \\
\hline Examination & function & & & & & less \\
\hline \multicolumn{7}{|l|}{ (MMSE) } \\
\hline Immediate Story & Logical memory & $\mathrm{IR}=$ correctly & 11 & \pm 3.94 & $3-19$ & Norm $=6.0$ \\
\hline \multirow[t]{2}{*}{ Recall } & & recalled out of 25 & & & & (3.0) \\
\hline & & items & & & & \\
\hline Delayed Story & & DRPIR $=\%$ of IR & 84 & \pm 2 ver. 57 & $14-180$ & Delayed \\
\hline \multirow[t]{3}{*}{ Recall } & & correctly recalled & & & & recall $+\%$ of \\
\hline & & after 20 minutes & & & & Immediate \\
\hline & & & & & & Recall. Norm \\
\hline
\end{tabular}




\begin{tabular}{|c|c|c|c|c|c|c|}
\hline National Adult & Estimated pre-morbid & Number of errors & 11 & \pm 7.19 & $0-36$ & \\
\hline Reading Test & intelligence & (out of 50 ) & & & & \\
\hline \multirow[t]{3}{*}{ (NART) } & & converted to IQ & & & & \\
\hline & & score & 116 & \pm 9.08 & $86-129$ & Average $=$ \\
\hline & & & & & & 100 \\
\hline Trail Making Test A & Visual attention & Speed in seconds & 32.00 & \pm 9.28 & $20.57-67.23$ & Norm = 100 \\
\hline (TMT A) & & to complete TMT B & & & & seconds \\
\hline Trail Making Test B & Task-switching & & 69.84 & \pm 28.10 & $28.73-162$ & Norm = 280 \\
\hline (TMT B & & & & & & seconds \\
\hline Digit Span & Verbal working memory & Number of full & 7 & \pm 1.14 & $5-8$ & Norm $=6 \pm 1$ \\
\hline Forwards (DS/F) & capacity (out of 8 ) & strings of digits & & & & \\
\hline
\end{tabular}


without error

\begin{tabular}{|c|c|c|c|c|c|c|}
\hline Digit Span & Cognitive processing & & 5 & \pm 1.05 & $3-7$ & Norm $=5 \pm 1$ \\
\hline Backwards (DS/B) & (out of 7) & & & & & \\
\hline Symbol Digit & Perceptual/processing & Number of correct & 44 & \pm 9.95 & $16-66$ & Norm $=37$ \\
\hline Modalities Test & speed and fluid & responses in 90 & & & & \\
\hline (SDMT) & cognitive ability & seconds & & & & \\
\hline
\end{tabular}


Table 2. Demographic and background characteristics

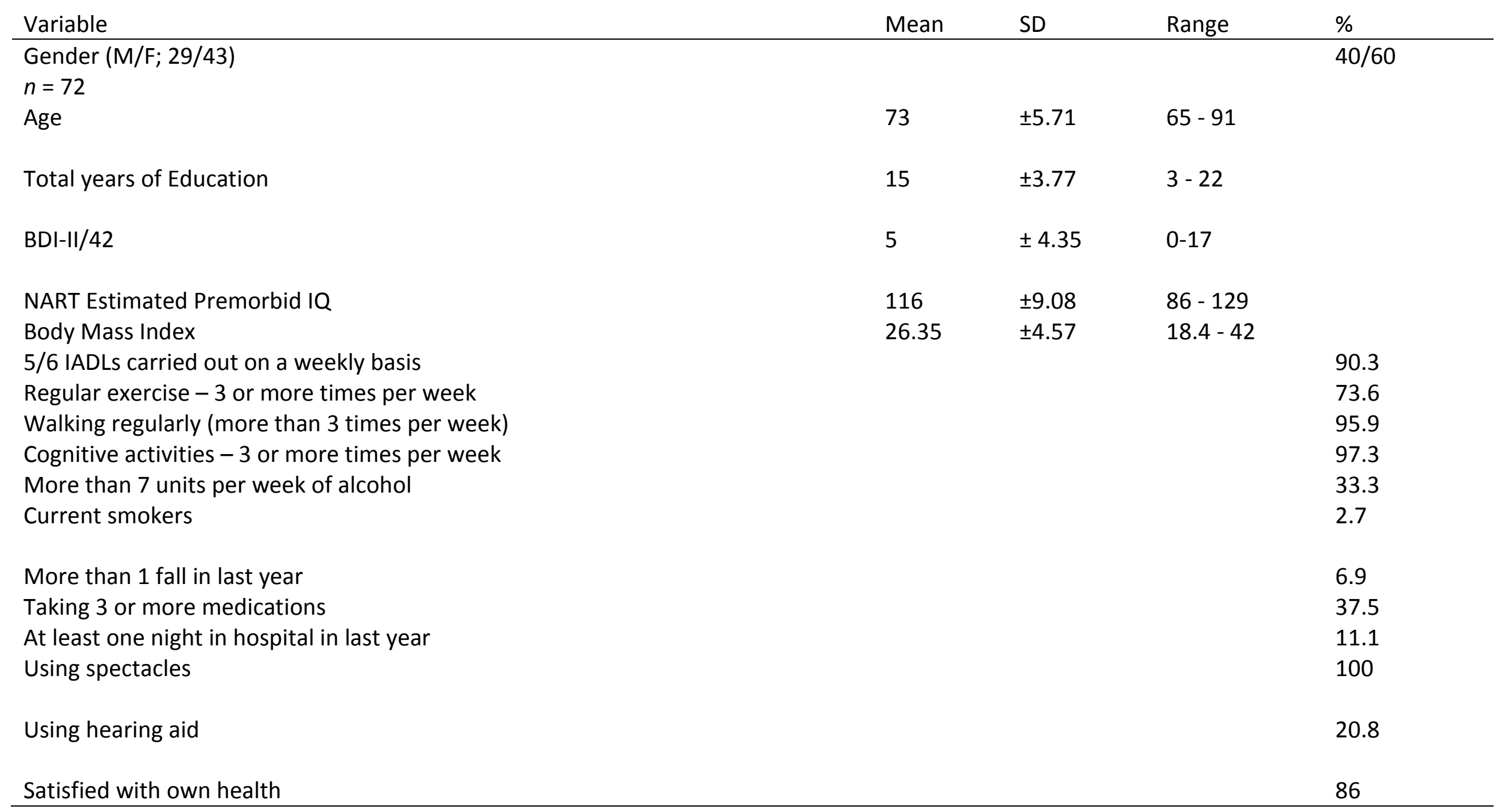


Table 3. Performance outcome measures (Gait variability (CV), Velocity, Correct Cognitive Responses) in Single and

Dual-task conditions (Prioritizing Walking, Prioritizing Counting)

\begin{tabular}{|c|c|c|c|c|}
\hline Condition & $\begin{array}{l}\text { Cognitive } \\
\text { Load }\end{array}$ & $\begin{array}{l}\text { Step-time } \\
\text { variability (CV) }\end{array}$ & Velocity & CCR \\
\hline ST Walking (Own & $\mathrm{N} / \mathrm{A}$ & $0.035 \pm(0.030)$ & $1.191 \pm(0.24)$ & $\mathrm{N} / \mathrm{A}$ \\
\hline \multicolumn{5}{|l|}{ Pace) } \\
\hline \multirow[t]{2}{*}{ ST Counting } & Serial 3s & $\mathrm{N} / \mathrm{A}$ & $N / A$ & $0.395 \pm(.162)$ \\
\hline & Serial 7s & $\mathrm{N} / \mathrm{A}$ & N/A & $0.249 \pm(.139)$ \\
\hline $\mathrm{DT}(\mathrm{PW})$ & Serial 3s & $0.049 \pm(0.047)$ & $1.07 \pm(0.26)$ & $0.470 \pm(.151)$ \\
\hline DTC/DTB & Serial 3s & $-0.013 \pm(0.043)$ & $0.126 \pm(0.173)$ & $-0.075 \pm(0.169)$ \\
\hline
\end{tabular}




\begin{tabular}{|c|c|c|c|c|}
\hline $\mathrm{DT}(\mathrm{PW})$ & Serial 7s & $0.051 \pm(0.049)$ & $1.09 \pm(0.29)$ & $0.334 \pm(.153)$ \\
\hline DTC/DTB & Serial 7s & $-0.016 \pm(0.041)$ & $0.186 \pm(0.218)$ & $-0.085 \pm(0.150)$ \\
\hline $\mathrm{DT}(\mathrm{PC})$ & Serial 3s & $0.052 \pm(0.049)$ & $0.99 \pm(0.28)$ & $0.535 \pm(.179)$ \\
\hline DTC/DTB & Serial 3s & $-0.017 \pm 0(.047)$ & $0.198 \pm(0.246)$ & $-0.139 \pm(0.152)$ \\
\hline $\mathrm{DT}(\mathrm{PC})$ & Serial 7s & $0.085 \pm(0.88)$ & $0.897 \pm(0.28)$ & $0.270 \pm(.166)$ \\
\hline DTC/DTB & Serial 7s & $-0.050 \pm(0.080)$ & $0.294 \pm(0.291)$ & $-0.021(.164)$ \\
\hline
\end{tabular}


Note 1: Values denote mean $(\mathrm{M}) \pm$ Standard Deviation (SD) for Step-time variability Coefficient of Variation (CV), Velocity in $\mathrm{m} / \mathrm{s}$ and Correct Cognitive Responses (CCR) per second in Prioritizing Walking (PW) and Prioritizing Counting (PC) conditions.

Note 2: Dual-task benefit (DTB) shown in bold font

Note 2: For velocity and CCRs, as better performance leads to greater values, DTCs are indicated by positive (ST-DT) values. For CCRs, DTBs are indicated by a negative (ST-DT) value. For step-time CV, as increased variability means less steady gait, DTCs are indicated by a negative (ST-DT) value. 
Table 4. Significant Effects*, Interactions** and differences*** results-Gait and Cognitive Outcomes

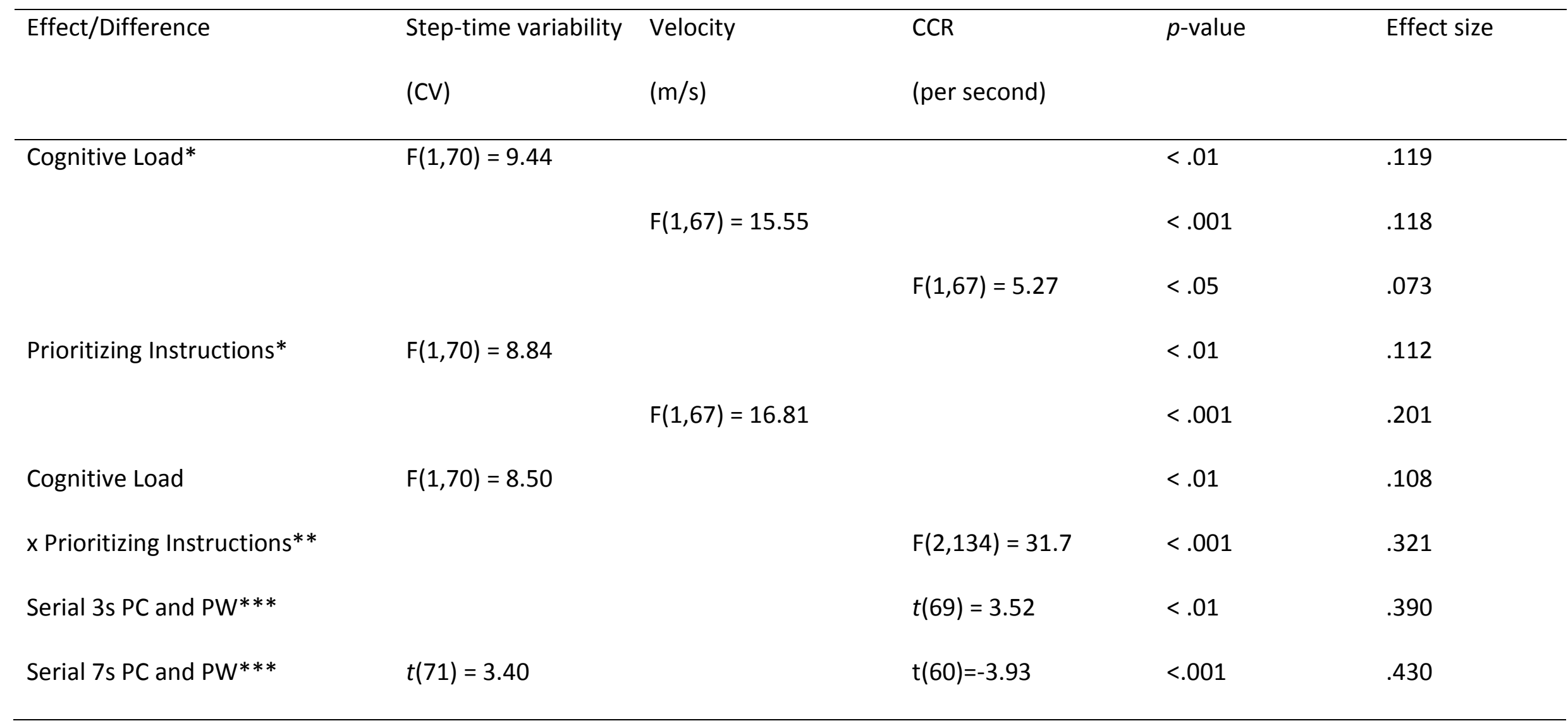

Levels of significance are at 0.05 and effect sizes are $\eta^{2}$ or $r$ 